Bundesgesundheitsbl 2018 $\cdot 61: 495-498$ https://doi.org/10.1007/s00103-018-2732-0 Online publiziert: 10. April 2018

(C) Springer-Verlag GmbH Deutschland, ein Teil von Springer Nature 2018

CrossMark

Lothar H. Wieler ${ }^{1}$ Karl Broich ${ }^{2}$

${ }^{1}$ Robert Koch-Institut, Berlin, Deutschland

${ }^{2}$ Bundesinstitut für Arzneimittel und Medizinprodukte, Bonn, Deutschland

\title{
Antibiotikaresistenzen: Große Herausforderungen erfordern komplexe Lösungen
}

Aktionspläne vor und auch Befragungen in der Bevölkerung belegen, dass eine wachsende Anzahl der Menschen sich der Bedrohung durch antibiotikaresistente Bakterien bewusst ist. Deutschland hat 2011 die Deutsche Antibiotika-Resistenzstrategie (DART) vorgestellt und diese mit DART 2020 im Mai 2015 weiterentwickelt. Da die Thematik der Entstehung und Verbreitung von Antibiotikaresistenzen vielschichtig und komplex ist und die Beherrschung des Problems die koordinierte Zusammenarbeit auf verschiedenen gesellschaftlichen Ebenen erfordert, stellt die Umsetzung dieser Strategie jedoch eine große Herausforderung dar.

Antibiotikaresistente Bakterien sind nicht einfach das Resultat menschlichen Handelns - sie kommen in allen Habitaten, in denen Bakterien leben vor, also überall auf dieser Erde. In diesen natürlichen Standorten bietet eine Antibiotikaresistenz den jeweiligen Bakterien oft einen Überlebensvorteil gegenüber anderen Bakterien. Dieser evolutionäre Vorteil wird seit den 1940er-Jahren jedoch durch den Menschen in hohem Maße selektioniert, und zwar durch den breiten Einsatz von Antibiotika. Je mehr Antibiotika verwendet werden, desto größer ist der Selektionsdruck auf jene Bakterien, die mit diesen Antibiotika in Kontakt kommen und desto häufiger kommt es zur Entstehung und Verbreitung von Resistenzen. Bakterien finden sich jedoch nicht nur in der Umwelt. Tatsächlich sind Menschen sowie alle anderen Lebewesen dicht mit Bakterien besiedelt - der menschliche Körper weist sogar etwa 15mal mehr bakterielle als eigene Zellen auf.
Der Selektionsdruck auf Bakterien wird zusätzlich dadurch gesteigert, dass Antibiotika nicht nur in der Humanmedizin, sondern auch in der Tiermedizin eingesetzt werden. Der Einsatz folgt nicht immer ausschließlich dem genuinen Ziel der Infektionstherapie oder im Falle operativer Eingriffe der Infektionsprophylaxe: So werden Antibiotika oftmals angewendet, obwohl die zugrunde liegenden Krankheiten dies nicht erfordern, oder sie werden in manchen Teilen der Welt als Wachstumsförderer in der Tiermast eingesetzt.

Dies ist problematisch, denn jede Anwendung von Antibiotika fördert die Selektion antibiotikaresistenter Bakterien. Wo Antibiotika besonders häufig und ungezielt eingesetzt werden, beschleunigt sich dieser Prozess weiter. Die Zunahme und Verbreitung antibiotikaresistenter Bakterien bedeutet gleichzeitig, dass es $\mathrm{zu}$ einer Anreicherung von genetischer Information kommt, die Antibiotikaresistenzmechanismen kodiert. Viele dieser genetischen Informationen sind aufGenomabschnitten lokalisiert, diehorizontal zwischen Bakterien übertragen werden können. Und diese biologischen Vorgänge finden in jedem Habitat statt, in dem Bakterien leben, d.h. im Boden, im Wasser und natürlich auch in der Tierwelt und im Menschen.

Wenn Sie diese Zusammenhänge berücksichtigen, liegen die beiden wichtigsten Grundsätze zur Eindämmung antibiotikaresistenter Bakterien auf der Hand: 1) Die Häufigkeit von Infektionen reduzieren und 2) Antibiotika nur dann einzusetzen, wenn sie wirklich vonnöten sind. Nur die konsequente Umsetzung 
dieser beiden Ziele kann den Prozess der Selektion und Anreicherung antibiotikaresistenter Bakterien und des „Resistoms“, also der Erbinformation für Resistenzen, verlangsamen. Die Entwicklung neuer Wirkstoffe ist ebenfalls wichtig - aber neue Wirkstoffe allein lösen das zentrale Problem nicht. Im Gegenteil sprechen alle uns bekannten biologischen Erkenntnisse dafür, dass sich auch gegen neue Antibiotika schnell Resistenzen bilden. Neue Wirkstoffe werden deshalb v. a. bei therapeutischen Lücken notwendig, die durch den Einsatz von alten Wirkstoffen geschaffen wurden. Letztlich wird diese Lücke aber immer nur so lange zu schließen sein, bis neue Resistenzmechanismen entstehen. Darum muss auch für neue Wirkstoffe gelten, dass sie nur dann zum Einsatz kommen dürfen, wenn dies medizinisch klar indiziert ist und eine gezielte Therapie möglich ist. Neue Wirkstoffe verschaffen uns also nur zusätzliche Zeit, aber sie lösen nicht das grundsätzliche Problem der Entstehung von Resistenzen.

Folgerichtig benennt der Globale Aktionsplan der WHO fünf strategische Ziele: Aufmerksamkeit und Wissen zum Thema erhöhen, eine Faktenbasis schaffen durch Einführung geeigneter Surveillance, die Infektionsprävention und -kontrolle stärken und den Einsatz von Antibiotika in Human- und Tiermedizin optimieren. Das fünfte Ziel adressiert Forschungsaspekte im Hinblick auf Diagnostika, Alternativen zu Antibiotika sowie die Entwicklung neuer Wirkstof$f e$. Dieser Logik folgt das vorliegende Themenheft: In erster Linie erfolgt eine Problembeschreibung samt Erörterung prinzipieller Lösungswege. Ziel ist es also nicht, die molekularen Mechanismen bakterieller Resistenz gegenüber Antibiotika oder eine infektionsepidemiologische Bedeutung einzelner bakterieller Erreger zu vermitteln. Vielmehr möchte das vorliegende Themenheft einen Überblick geben über die Vielzahl der Aktivitäten und Konzepte, die alle für sich und hoffentlich in steigender Intensität miteinander vernetzt diese umfangreiche Herausforderung annehmen: das Risiko, das von antibiotikaresistenten Bakterien ausgeht, zu reduzieren und somit die
Gesundheit von Mensch und Tier besser zu schützen.

Die ersten drei Beiträge haben den Schwerpunkt „Bewusstsein und Verstehen fördern, Wissen erweitern“. Die Beiträge sprechen das One-Health-Konzept an, welches auf die Untrennbarkeit der Gesundheit von Mensch und Tier mit einer gesunden Umwelt fokussiert. Tatsächlich ist die Antibiotikaresistenz das Paradebeispiel für One-Health-Ansätze. Antão und Wagner-Ahlfs beschreiben zunächst beispielhaft, warum Antibiotikaresistenzen im Fokus des Gesundheitsschutzes stehen müssen. Dann wird anschaulich die Komplexität dieses Problems erörtert. Eine Lösung ist nur durch Einbindung der Akteure „Policy, Prevention, Prescribers, Pharmacy, Patients“ - das sog. 5-P-Konzept - möglich. Alle genannten Akteure haben eine Verantwortung, der sie gemeinsam nachkommen müssen. Nicht nur behandelnde Ärzte und Tierärzte oder die pharmazeutische Industrie tragen Verantwortung, sondern auch die Patienten. Um dieser Verantwortung gerecht zu werden, müssen aber alle betroffenen Akteure hinreichend über die Auswirkungen des Antibiotikaeinsatzes informiert sein - eine Aussage, die die Autoren in ihrem Beitrag unterstreichen. In Deutschland widmet sich diesem Ansatz das beispielhaft genannte Forschungskonsortium InfectControl 2020 in herausragender Weise.

Abu-Sin et al. widmen sich den nationalen und globalen Ansätzen. In einer umfassenden Übersicht benennen und erläutern sie Bedeutung und Aufgaben zunächst der supranationalen Einrichtungen, bis sie schließlich auf nationale Ressortforschungsinstitute fokussieren. Hier wird deutlich, wie viele Aktivitäten gerade in den letzten Jahren initiiert wurden und wie anspruchsvoll eine zielführende Koordinierung derselben ist. So wird auch klar, wie die DART 2020 als zentrales Bindeglied zwischen Deutschland und den internationalen Institutionen fungiert. Entsprechend liegt das Fazit auf der Hand: Erfolge sind nur zu erreichen, wenn die globalen und nationalen Strategien konsequent umgesetzt werden und eine gute Koordination und ein intensiver Austausch auf nationaler und internationaler Ebene erfolgen. Die dritte Voraussetzung ist nicht weniger anspruchsvoll: Die zahlreichen Aktivitäten müssen selbstverständlich begleitend evaluiert werden. Es ist sehr wichtig, die Evidenz für Maßnahmen bei der Bekämpfung von Antibiotikaresistenz zu stärken - damit steigt die Qualität der Empfehlungen und deren Akzeptanz.

Den Übertragungswegen zwischen Tier und Mensch widmet sich der Beitrag von Tenhagen et al. Die Autoren betonen, dass die Übertragung von antibiotikaresistenten Bakterien keine Einbahnstraße ist - auch Menschen können Infektionsquelle für Tiere sein. Sie unterscheiden drei prinzipielle Wege der Übertragung: über Kontakt, über Lebensmittel tierischer Herkunft sowie über die Umwelt. Die quantitative Bedeutung dieser Wege ist bisher aber noch unklar - hier besteht Forschungsbedarf.

Unter dem Schwerpunkt „Surveillance und Interventionen verbessern" beschreiben zunächst Noll et al. bestehende Systeme in der Tier- und Humanmedizin. Die Unterschiede sind sehr deutlich und es überrascht aufgrund der sehr vielfältigen Tierhaltungsbedingungen nicht, dass die Systeme in der Humanmedizin häufig spezifischer sind. Interessant ist dabei jedoch ein Ansatz, der mit dem 16. Gesetz zur Änderung des Arzneimittelgesetzes im Jahr 2014 für Halter von Masttieren in Deutschland die Verpflichtung einführte, angewendete Antibiotika zu melden, um eine individuelle betriebliche Therapiehäufigkeit zu ermitteln. So können die Therapiehäufigkeiten einzelner Tierhalter miteinander verglichen werden. Liegt der Tierhalter bei dem Einsatz von Antibiotika in einem hohen Bereich, kann gezielt nachgeforscht und interveniert werden. Eine derart weitgehende Intervention von gesetzlicher Seite ist in der Humanmedizin in Deutschland nicht gegeben, hier obliegt die Bewertung des Antibiotikaverbrauchs der Eigenverantwortlichkeit der Leiter der jeweiligen Einrichtungen.

Eine ganz andere Problematik beleuchten Westphal-Settele und Mitautoren. Sie diskutieren die Bedeutung der Umwelt als Reservoir für antibiotikaresistente Bakterien. Ausgehend von aktuellen Antibiotikaverbrauchs- bzw. 
Abgabemengen werden die Eintragswege von Arzneimitteln in die Umwelt beschrieben und durch den Nachweis von Antibiotikarückständen belegt. Es wird der Umfang der Risiken einer Übertragung von antibiotikaresistenten Bakterien und deren Resistoms aus der Umwelt auf Mensch und Tier erläutert. Seit Mitte der 1970er-Jahre liegen Hinweise auf eine Verbindung der Resistenzen aus der Umwelt zu denen im klinischen Umfeld vor, aber es fehlen nach wie vor ausreichend belastbare Daten.

Dohle und Co-Autoren berichten sehr konkret über das Management eines Ausbruchs mit Klebsiella pneumoniae in einer Rehabilitationsklinik. Dieser Report belegt einmal mehr, wie wichtig eine Surveillance im stationären Bereich ist, denn nur das rechtzeitige Erkennen eines Ausbruchs führt zur Einleitung von effektiven Gegenmaßnahmen. Was diesen Report besonders interessant macht, ist der Ausbruch in einer Rehabilitationsklinik. Nosokomiale Ausbrüche in Akutkliniken sind bereits klar im Bewusstsein von Ärzten und medizinischem Fachpersonal verankert, aber Geschehen in Rehabilitationseinrichtungen stehen bisher weniger im Fokus. Dabei ist es eigentlich wenig verwunderlich, dass aufgrund der längeren Aufenthaltsdauer und insbesondere im Falle von Katheterisierungen, wie sie in einer Neurorehabilitation üblich sind, solche Einrichtungen ein hohes Risiko nosokomialer Infektionen beherbergen. Daher sind diese Einrichtungen in $₫ 23$ IfSG auch explizit berücksichtigt.

Die beiden folgenden Beiträge von Mielke sowie von Brunkhorst et al. haben den Aspekt der wissenschaftlich begründeten Infektionsprävention und -kontrolle - auch, aber nicht nur bei Vorliegen antibiotikaresistenter Erreger - im Fokus. Diese ist möglich, wenn valide Daten zu Art und Umfang des Problems vorliegen. Besonders der Beitrag von Mielke spricht darüber hinaus eine ebenso simple wie überzeugende Tatsache an: Jede verhinderte Infektion reduziert den Einsatz von Antibiotika und dient damit auch der Eindämmung von Antibiotikaresistenzen. Der Beitrag ist argumentativ ebenso stringent wie überzeugend und erweitert die auf die Bekämpfung antibiotikaresistenter
Bakterien fokussierten Aktivitäten um die breiter angelegte generelle Infektionsprävention und Patientensicherheit. Brunkhorst et al. arbeiten sehr überzeugend die Problematik des Fehlens valider Daten zur Epidemiologie der Sepsis heraus, wodurch natürlich auch das Fehlen flächendeckender Interventionsstrategien erklärbar ist. Die hohe Sterblichkeit bei Sepsis kann jedoch nur gesenkt werden, wenn neue Ansätze in der Früherkennung erforscht werden. Die Autoren fordern deshalb eine angemessene Blutkulturabnahmerate sowie eine hohe präanalytische Qualität als Grundlage der Qualitätssicherung insbesondere im Bereich der nosokomial erworbenen Sepsis.

Der vorletzte Schwerpunkt des Themenheftes behandelt den optimierten Einsatz von Antibiotika. Abele-Horn und Co-Autoren arbeiten sehr überzeugend jene vier Bereiche heraus, deren Optimierung und Vernetzung zu einer signifikanten Verbesserung des rationalen Antibiotikaeinsatzes führen würden: gezielte sachgerechte Diagnostik, Beachtung jeweils aktualisierter Leitlinien, restriktive Maßnahmen und gute Kommunikation. Die konsequente Umsetzung bereits verabschiedeter qualitativ hochwertiger Leitlinien würde viel bewegen. Ohne ihre Argumente vorwegzunehmen, sei hier auf eine folgenreiche Fehlsteuerung der Infektionsmedizin hingewiesen: die mangelnden Anreize für die Mitarbeit von Kollegen in Kommissionen und Fachgesellschaften, die hochwertige Leitlinien zur Diagnostik und zum Antibiotikaeinsatz verabschieden. Hier muss die biomedizinische Wissenschaftsgemeinschaft endlich umdenken, denn letztlich ist ihr Forschungsauftrag in der Verbesserung der Gesundheit der Bevölkerung begründet. Die einseitige Fokussierung von Leistungsmessung anhand hoher Impactfaktoren und umfangreicher Drittmitteleinwerbungen stehen nicht zwingend in positiver Assoziation zu diesem Ziel.

Eines der von Abele-Horn und CoAutoren angesprochenen Themen - Antibiotic Stewardship - führt W. Kern in seinem Beitrag weiter aus. Eingehend beschreibt er die Entwicklung in den letzten Jahren und folgert daraus konkrete Akti- vitäten. Kern sieht die zielgerichtete Vernetzung verschiedener Akteure auch als Schlüssel zum Erfolg: die Schulung und Fortbildung von ärztlichem Personal und Apothekern und die Etablierung von Antibiotic-Stewardship-Teams sind ebenso wichtig wie die Einrichtung infektiologischer Konsiliardienste im Krankenhaus. Die Verordnungsqualität ist dabei von besonderer Güte, wenn die mikrobiologische Expertise umfänglich eingebunden wird. Er lässt auch keinen Zweifel aufkommen, dass die Schnittstellen zwischen stationärer und ambulanter Medizin verbessert werden müssen. Da rund $85 \%$ aller Antibiotikaverschreibungen in ambulanten Praxen erfolgen, ist diese Forderung nur folgerichtig.

Einen eher selten diskutierten Aspekt der Problematik beleuchten Littmann und Buyx, nämlich deren ethische Implikationen. Die ethischen Konsequenzen der Antibiotikaresistenz lassen sich grob in zwei Kategorien einteilen: ethische Herausforderungen, die durch Antibiotikaresistenz verstärkt werden, und ethische Herausforderungen, die durch Antibiotikaresistenz entstehen. Littmann und Buyx beschränken ihre Ausführungen auf das Problem der Verteilungsgerechtigkeit des Antibiotikaeinsatzes, konkret die ethischen Implikationen des rationalen Antibiotikaeinsatzes und die gerechte Risikoverteilung. Der WHO-Aktionsplan definiert Antibiotika als „öffentliches Gut", nach Ansicht der Autoren eine nichtzutreffende Kategorisierung, denn diese Definition basiert auf zwei Grundannahmen: Kein Mensch kann von einem öffentlichen Gut ausgeschlossen werden und der Gebrauch des öffentlichen Guts hat keine nachteiligen Auswirkungen auf Mitmenschen. Beides trifft aber auf Antibiotika nicht zu: Tatsächlich stehen weltweit sehr vielen Menschen die notwendigen bzw. sachgerecht indizierten Antibiotika wegen eines mangelnden $\mathrm{Zu}$ gangs zu medizinischer Versorgung nicht zur Verfügung und außerdem führt die Anwendung ja zur Anreicherung von Resistenzen und hat damit einen negativen Einfluss auf die Wirkung der Antibiotika. Würde sich der rationale Antibiotikaeinsatz lediglich auf die Eliminierung offensichtlich unangemessener Verschreibungen beziehen, gäbe es kaum ethische 
Implikationen. Aber ein sparsamer Einsatz von Antibiotika wirft letztlich auch die Frage auf, wie groß der Nutzen für den Patienten mindestens sein muss, um eine Therapie zu rechtfertigen. Die Autoren fordern daher eine klare Definition des Begriffs „rationaler Antibiotikaeinsatz" unter Einbeziehung der Risiken und Nutzen. $\mathrm{Ob}$ sich alle Mediziner dieser Forderung anschließen können?

$\mathrm{Zu}$ guter Letzt darf auch die bereits erwähnte Wirkstoffforschung nicht fehlen. Der Beitrag von Kloß und Gerbach fasst an ausgewählten Beispielen die Herausforderungen zusammen, nicht ohne darauf einzugehen, dass auch diese Forschung die Bildung von Resistenzen minimieren muss. Sie fokussieren auf Antibiotika sowie Alternativen wie Antivirulenz- und Phagentherapie und Immunmodulatoren. Sie fordern wegen der gesamtgesellschaftlichen Bedeutung Forschungsinfrastrukturen und öffentlichprivate Partnerschaften zu stärken, regulatorische Hürden abzubauen und Innovationen für die Antibiotikatherapie mit Hochdruck voranzutreiben.

Sehr geehrte Leser, die Bandbreite der Beiträge zum Themenheft „Antibiotikaresistenzen, eine komplexe gesamtgesellschaftliche Herausforderung" belegt nachdrücklich das gewaltige Ausmaß der Herausforderungen. Wenn Sie die Beiträge lesen, werden Sie einen roten Faden finden: Alle Autoren verdeutlichen, dass die Herausforderungen nur dann bewältigt werden können, wenn viele Akteure ihr Wissen teilen, sich miteinander vernetzen und evidenzbasiert handeln. Dieser Imperativ ist allen Kennern der Naturwissenschaften hinlänglich bekannt - hoffen wir, dass der Appell unserer Autoren auf fruchtbaren Boden fällt. Wir sind allen Autoren sehr dankbar - Ihnen wünschen wir eine bereichernde Lektüre.
Mit freundlichen Grüßen

Ihre

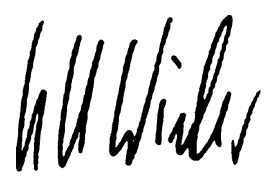

Lothar H. Wieler

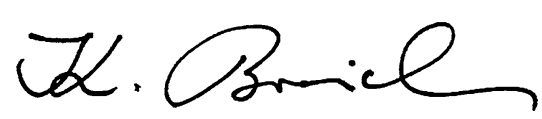

Karl Broich

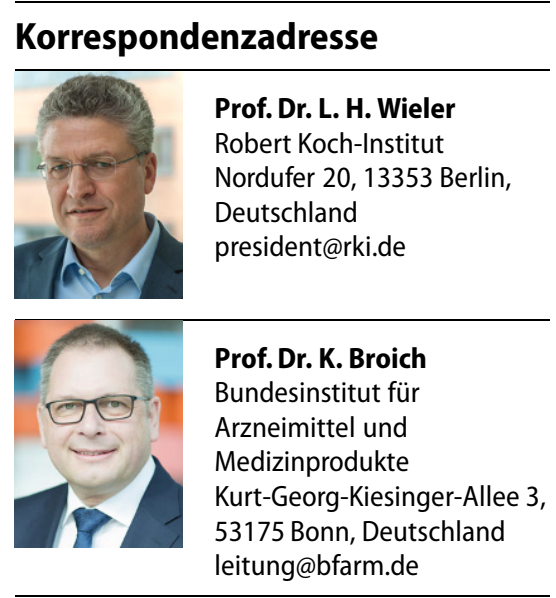

Interessenkonflikt. L.H. Wieler und K. Broich geben an, dass kein Interessenkonflikt besteht. 